N. van Quê and G. E. Reyes, Smooth functors and synthetic calculus, in the L. E. J. Brouwer Centenary Symposium A. S. Troelstra and D. van Dalen (eds.), North-Holland, 1982, 377-395.

G. E. Reyes, Synthetic reasoning and variable sets (Models for synthetic differential geometry), in Categories in Continuum Physics, Lecture Notes in Math. 1174, Springer-Verlag, 1986, 69-82.

- Analyse dans les topos lisses, Cahiers de Top. et de Géom. Difierentielle 22-2 (1981).

J-C. Tougeron (1972), Idéaux de fonctions différentiables, Springer, Berlin 1972.

MATHEMATISCH INSTITUUT

UNIVERSITEIT VAN AMSTERDAM

Roeterstraat 15

1018 WB Amsterd

DÉPARTEMENT DE MATHEMATIQUES

UNIVERSITÉ DE MONTREAL

Case postale 6128,
Canada $\mathrm{H} 3 \mathrm{C} .3 \mathrm{~J} 7$

Received 23 May 1985;

in revised form 26 May 1986

\section{Pointwise limits of subsequences and $\Sigma_{2}^{1}$ sets}

by

Howard Becker* (Columbia, S. C.)

Abstract. The following representation theorem for $\Sigma_{2}^{1}$ subsets of the space $C[0,1]$ is proved, and some applications of it are given. For any $\Sigma_{2}^{1}$ set $S \subset C[0,1]$, there exists a sequence $\left\langle f_{i}\right\rangle$ of continuous functions such that $S$ is the set of all continuous pointwise limits of subsequences of $\left\langle f_{i}\right\rangle$.

§1. The Main Theorem. A Polish space is a topological space homeomorphic to a separable complete metric space. In this paper all spaces are Polish. For any space $X$, let $X^{\omega}$ denote the topological product of countably many copies of $X$. Let $C$ be the space $C[0,1]$ of continuous real-valued functions on the unit interval, with the uniform metric. This paper is mainly concerned with the two spaces $C$ and $C^{\omega}$. The elements of $C^{\omega}$ are sequences of functions; our notation for these sequences is $\left\langle f_{i}\right\rangle,\left\langle g_{i}\right\rangle, \ldots$

A pointset is $\Sigma_{1}^{1}$ if it is the projection of a Borel set (in some product space). A $\Pi_{n}^{1}$ set is the complement of a $\Sigma_{n}^{1}$ set and a $\Sigma_{n+1}^{1}$ set is the projection of a $\Pi_{n}^{1}$ set (in some product space). A set is $\boldsymbol{\Delta}_{n}^{1}$ if it is both $\boldsymbol{\Sigma}_{n}^{1}$ and $\boldsymbol{\Pi}_{n}^{\mathbf{1}}$. This is the logicians' notation - the classical names for $\Sigma_{1}^{1}, \Pi_{1}^{1}, \Sigma_{2}^{1}, \Pi_{2}^{1}, \Sigma_{3}^{1}, \ldots$ are $A$ (analytic), CA (coanalytic), PCA, CPCA, PCPCA, ... Any two uncountable Polish spaces are Borel isomorphic, and these classes are all preserved under Borel isomorphism, so as far as the abstract theory of $\Sigma_{n}^{1}$ sets is concerned, there is only one space. Hence descriptive set theory, the study of pointclasses such as $\Sigma_{n}^{1}$, is frequently presented in the context of one fixed space, $\omega^{\omega}$ (Baire space), where $\omega$ is the natural numbers with the discrete topology. A good reference for descriptive set theory is Moschovakis [12], whose notation and terminology will be used in this paper.

1.1. Definition. Let $\left\langle f_{i}\right\rangle \in C^{\omega}$. Then $A_{\left\langle f_{i}\right\rangle}$ denotes the following subset of $C$ :

$\left\{h \in C\right.$ : there is a subsequence of $\left\langle f_{i}\right\rangle$ which converges pointwise to $\left.h\right\}$.

Note that for any $\left\langle f_{i}\right\rangle$, the pointset $A_{\left\langle f_{i}\right\rangle}$ is $\Sigma_{2}^{1}$, uniformly. (This is proved by the methods of $[12,1 \mathrm{C}$ and $1 \mathrm{E}]$.) The main theorem of this paper is the converse - every $\Sigma_{2}^{1}$ set can be represented in this manner.

* Research partially supported by NSF Grant MCS 82-11328. 
1.2. THeOREM. For any $S \subset C$, if $S$ is $\Sigma_{2}^{1}$ then there exists an $\left\langle f_{i}\right\rangle$ in $C^{(3)}$ such that $S=A_{\left\langle f_{i}\right\rangle}$.

Several representation theorems for $\Sigma_{1}^{1}$ sets have appeared in the literature. One example of such a theorem is due to Poprougénko [14], who showed that every $\Sigma_{1}^{1}$ set of real numbers is the range of a derivative. Others can be found in the following references: Bagemihl-Mc Millan [1], Kaufman [5], [6], Lorentz-Zeller [10], Nishiura [13]. I would like to thank Alexander Kechris for bringing these theorems to my attention. There are also several theorems which give representations of $\Sigma_{n}^{1}$ sets in terms either of concepts from the abstract theory of $\Sigma_{n}^{1}$ sets, or concepts from mathematical logic (see Moschovakis [12]). Theorem 1.2 is the only example I know of which gives a representation of $\Sigma_{2}^{1}$ sets in terms of concepts from analysis or topology. I know of no examples for $\Sigma_{n}^{1}$ when $n \geqslant 3$.

This paper is organized as follows. $\S 2$ contains some corollaries to the theorem. It also contains a number of miscellaneous remarks and questions. The rest of the paper is devoted to proving Theorem 1.2. In $\S 3$ we prove 1.2 for $\boldsymbol{\Pi}_{1}^{1}$ sets, that is, we show that for any $\Pi_{1}^{1}$ set $P \subset C$, there is an $\left\langle f_{i}\right\rangle$ such that $P=A_{\left\langle f_{i}\right\rangle} . \ln \S 4$ we show how to transfer this result from $\Pi_{1}^{1}$ to $\Sigma_{2}^{1}$, and thereby complete the proof.

§ 2. Corollaries, examples, remarks, questions, etc.

2.1. Let $D$ be any dense subset of $C$. It is obvious that given any $\left\langle g_{i}\right\rangle \in C^{(\prime)}$, there is an $\left\langle h_{i}\right\rangle \in D^{\omega}$ such that $A_{\langle g i\rangle}=A_{\left\langle h_{i}\right\rangle}$.

Hence Theorem 1.2 can be strengthened by requiring the $f_{i}$ 's to be in $D$. For example, the $f_{i}$ 's can always be taken to be polynomials, or to be piecewise-linear.

2.2. Most subsets of $C$ (in the sense of cardinality) are not $\Sigma_{2}^{1}$, but as a practical matter, virtually every pointset that would ever occur in ordinary mathematics is. To cite one familiar example, the set of differentiable functions is $\boldsymbol{\Pi}_{1}^{1}$, hence $\Sigma_{2}^{1}$. (The set of differentiable functions is not Borel, by a theorem of Mazurkiewicz [11] see also Kechris -Woodin [8].) The simplest example known to me of a set which is not $\Sigma_{2}^{1}$, and hence to which the Main Theorem is not applicable, is the set of functions satisfying the Mean Value Theorem, that is,

$\{f \in C:$ for any $a, b$, if $0 \leqslant a<b \leqslant 1$ then there is a $c$ such that $a<c<b, f$ is differentiable at $c$, and $\left.f^{\prime}(c)=\frac{f(b)-f(a)}{b-a}\right\}$.

This set is clearly $\boldsymbol{\Pi}_{2}^{1}$; that it is not $\Sigma_{2}^{1}$ is an unpublished theorem of Woodin. (Incidentally, the set of functions satisfying Rolle's Theorem is $\Sigma_{1}^{1}$.)

2.3. Let $A \subset C^{\infty} \times C$ be the set $\left\{\left(\left\langle f_{i}\right\rangle, h\right): h \in A_{\left\langle S_{i}\right\rangle}\right\}$. Then $A$ is a universal set for $\Sigma_{2}^{1} \uparrow C$, that is, $A$ is $\Sigma_{2}^{1}$ and every $\Sigma_{2}^{1}$ subset of $C$ is a vertical section of $A$. (It is a classical theorem that for any $n$ and any uncountable Polish spaces $X$ and $Y$, there is a $U \subset X \times Y$ which is a universal set for $\Sigma_{n}^{1} \uparrow Y$, and similarly for $\Pi_{n}^{1}$ [12, 1D.1]. While the abstract theory tells us that universal sets exist, it leaves open the question of whether there are any examples of this phenomenon which arise naturally in analysis.) The representation theorems for $\Sigma_{1}^{1}$ mentioned in $\S 1$ also give natural examples of universal sets for $\Sigma_{1}^{1}$.

2.4. The Main Theorem holds uniformly. This means that if $U \subset \omega^{\omega} \times C$ is the canonical universal set for $\Sigma_{2}^{1} \uparrow C$ and $U_{x}=\{h \in C:(x, h) \in U\}$, then there is a recursive (hence continuous) function $G: \omega^{\omega} \rightarrow C^{\omega}$ such that for all $x \in \omega^{\omega}$, if we let $\left\langle f_{i}^{x}\right\rangle$ denote the sequence $G(x)$, then $U_{x}=A_{\left\langle f_{i}^{x}\right\rangle}$. It follows that for any $\Sigma_{2}^{1}$ set $S \subset X \times C$, there is a continuous function $G_{S}: X \rightarrow C^{\infty}$ with the above property. For details, see $[12,3 \mathrm{H}]$. The proof that the Main Theorem holds uniformly is implicit in the proof of the Main Theorem to be given in this paper.

2.5. Define two subsets $E$ and $F$ of $C^{\infty}$ as follows:

$$
\begin{aligned}
& E=\left\{\left\langle f_{i}\right\rangle \in C^{\omega}: \text { for every } h \in C, h \in A_{\left\langle f_{i}\right\rangle}\right\} . \\
& F=\left\{\left\langle f_{i}\right\rangle \in C^{\omega}: \text { there exists an } h \in C \text { such that } h \in A_{\left\langle f_{i}\right\rangle}\right\} .
\end{aligned}
$$

Clearly $E$ is $\boldsymbol{M}_{3}^{1}$ and $F$ is $\Sigma_{2}^{1}$. It follows from 2.4 that $E$ is complete $\Pi_{3}^{1}$, hence not $\Sigma_{3}^{1}$, and $F$ is complete $\Sigma_{2}^{1}$, hence not $\Pi_{2}^{1}$. Let $G$ be the set

$$
\left\{\left\langle f_{i}\right\rangle \in C^{\omega}:\left\langle f_{i}\right\rangle \text { converges pointwise and } \lim _{i \rightarrow \infty} f_{i} \text { is continuous }\right\} \text {. }
$$

$G$ is $\Pi_{1}^{1} . G$ is not $\Sigma_{1}^{1}$, since if it was then $F$ would also be $\Sigma_{1}^{1}$. (Assuming $\Pi_{1}^{1}$-determinacy, any set which is $\Pi_{1}^{1}$ and not $\Sigma_{1}^{1}$ is complete $\left.\Pi_{1}^{1}[12,7 D .3]\right]$; as one would expect, it is provable in $\mathrm{ZFC}$ that $G$ is complete $\Pi_{1}^{1}$.)

2.6. For $\left\langle f_{i}\right\rangle \in C^{\infty}$, let

$\hat{A}_{\left\langle f_{i}\right\rangle}=\{h \in C:$ there is a recursive-in- $h$ function $\alpha: \omega \rightarrow \omega, \alpha$ strictly increasing, such that the subsequence $\left\langle f_{a(i)}\right\rangle_{i \in \omega}$ converges pointwise to $h\}$.

For any $\left\langle f_{i}\right\rangle$, the pointset $\hat{A}_{\left\langle f_{i}\right\rangle}$ is $\Pi_{1}^{1}$, uniformly. It is implicit in the proof given in $\S 3$ that for any $P \subset C$, if $P$ is $\boldsymbol{\Pi}_{1}^{1}$ then there exists an $\left\langle f_{i}\right\rangle$ in $C^{\omega}$ such that $P=A_{\left\langle f_{i}\right\rangle}=\widehat{A}_{\left\langle f_{i}\right\rangle}$. Therefore if $\hat{A} \subset C^{\omega} \times C$ is defined to be $\left\{\left(\left\langle f_{i}\right\rangle, h\right): h \in \widehat{A}_{\left\langle f_{i}\right\rangle}\right\}$, then $\widehat{A}$ is a universal set for $\Pi_{1}^{1} \uparrow C$.

2.7. It is consistent with $\mathrm{ZF}+\mathrm{DC}$ that the binary relation

$$
B=\left\{\left(\left\langle f_{i}\right\rangle, h\right): h \notin A_{\left\langle f_{i}\right\rangle}\right\} \subset C^{\omega} \times C
$$

cannot be uniformized, that is, there is no choice function which assigns to each $\left\langle f_{i}\right\rangle$ in the domain of $B$, an $h$ such that $\left(\left\langle f_{i}\right\rangle, h\right) \in B$. It is consistent with ZFC that $B$ has no uniformization ordinal-definable from a real parameter. (Throughout Remark 2.7, a "real" is a subset of $\omega$.)

The first consistency result follows from the second by standard methods. Toward proving the second, let $R \subset 2^{\omega} \times 2^{\omega}$ be the binary relation $\{(x, y): y \notin L(x)\}$, where $L(x)$ is the constructible universe relativized to the real $x$, and consider the following proposition:

(*) $R$ has no uniformization which is ordinal-definable from a real. 
Since $R$ is $\Pi_{2}^{1}$, it follows from (*), the fact that $C$ is Borel isomorphic to $2^{\omega}$, and the uniform version of the Main Theorem (2.4), that $B$ has no uniformization ordinaldefinable from a real. The consistency of $(*)$ with ZFC is part of the folklore of set theory; however I have not been able to find it in print. Using some well-known facts about forcing, it is not hard to show that (*) holds in the model obtained by adding $s_{1}$ Cohen reals to $L$. For information on forcing and consistency proofs, see Jech [4]. The consistency results mentioned here are all variants of a theorem of Levy [9].

Of course it is also consistent with ZFC that $B$ does have an ordinal-definable uniformization. This is the case if $V=L$.

2.8. There are various types of pathological pointsets (e.g., nonmeasurable) which can be produced using the axiom of choice. Assuming $V=L$, these pathologies all exist at the second level of the projective hierarchy (see [12, 5A.8]); hence by 1.2 these pathologies can be sets of the form $A_{\left\langle f_{i}\right\rangle}$. Thus it is consistent with ZFC that there exists an $\left\langle f_{i}\right\rangle \in C^{\omega}$ such that all of the following hold:

(a) $A_{\left\langle f_{i}\right\rangle}$ is uncountable but has no perfect subset.

(b) $A_{\left\langle f_{i}\right\rangle}$ does not have the property of Baire.

(c) $A_{\left\langle f_{i}\right\rangle}$ is not measurable with respect to any nonatomic $\sigma$-finite Borel measure on $C$

On the other hand, it is also consistent that no $\Sigma_{2}^{1}$ set can exhibit such pathologies (see [4, p. 534 and p. 548]).

2.9. Let $E \subset C^{\omega}$ be as in 2.5, and let

$E_{1}=\left\{\left\langle f_{i}\right\rangle \in C^{\omega}\right.$ : there is exactly one $h \in C$ such that $\left.h \notin A_{\left\langle f_{i}\right\rangle}\right\}$.

By combining the proof that $E$ is not $\Sigma_{3}^{1}$ (2.5) with the method of Becker [2], we obtain the following theorem. Assuming $\Delta_{2}^{1}$-determinacy, $E$ and $E_{1}$ are a pair of disjoint $\Pi_{3}^{1}$ sets which cannot be separated by any $\Delta_{3}^{1}$ set. (If $V=L$, then every pair of disjoint $\boldsymbol{\Pi}_{3}^{1}$ sets can be separated by a $\Delta_{3}^{1}$ set $[12,5 \mathrm{~A} .3]$.)

2.10. Any $\boldsymbol{\Pi}_{1}^{1}$ set admits a $\boldsymbol{\Pi}_{1}^{1}$-norm. (See [12] for definitions and details.) It is the thesis of Kechris-Woodin [8] that natural examples of $\boldsymbol{M}_{1}^{3}$ sets will have natural $\Pi_{1}^{1}$-norms; they give an example of such a norm on the set of differentiable functions. For another example, consider the space $K(X)$ of compact subsets of $X$, with the Hausdorff metric, and the pointset in $K(X)$ of all countable compact sets. This is $\boldsymbol{\Pi}_{1}^{1}$ (and by a theorem of Hurewicz [3], is complete $\boldsymbol{\Pi}_{1}^{1}$ ). The Cantor-Bendixson rank is a $\boldsymbol{\Pi}_{\mathbf{1}}^{\mathbf{1}}$-norm on this set. An example more closely related to the topic of this paper, a natural $\boldsymbol{\Pi}_{1}^{1}$-norm on the $\boldsymbol{\Pi}_{1}^{1}$ set of pointwise convergent sequences, was discovered by Zalcwasser [17].

We wish to consider the same question for $\boldsymbol{\Pi}_{3}^{1}$. Assuming $\boldsymbol{\Delta}_{2}^{1}$-determinacy, every $\boldsymbol{\Pi}_{3}^{1}$ set admits a $\boldsymbol{\Pi}_{3}^{1}$-norm. (Assuming $V=L$, this is not so.) Consider the $\boldsymbol{\Pi}_{3}^{1}$ set $E \subset C^{\omega}$ of 2.5, and for the rest of Remark 2.10, assume $\Delta_{2}^{1}$-determinacy. Does $E$ have any natural $\boldsymbol{\Pi}_{3}^{1}$-norm? A positive answer to this (rather vague) open question would be of interest for two reasons. First, such a norm would constitute a hierarchical structure on the set $E$, and this structure might give useful information about $E$ or be used to prove theorems by transfinite induction. Second, since $E$ is a compiete $\boldsymbol{\Pi}_{3}^{1}$ set (2.5), the length of any $\boldsymbol{\Pi}_{3}^{1}$-norm on $E$ must be the ordinal $\delta_{3}^{1}$ [12, 4C.14]; hence a natural norm would give a description of this ordinal in terms of real analysis rather than of logic.

2.11. A Baire-1 function is, by definition, a pointwise limit of continuous functions. The set of Baire-1 functions does not form a Polish space in any natural way. However we can encode Baire-1 functions by elements of the space $C^{\omega}:\left\langle f_{i}\right\rangle$ encodes the pointwise limit of $\left\langle f_{i}\right\rangle$, if it exists. The set of codes is $\boldsymbol{\Pi}_{1}^{1}$, and the induced equivalence relation on the codes is also $\boldsymbol{M}_{1}^{1}$. Sets of Baire-1 functions correspond to invariant subsets of $C^{\omega}$, and we say that a set of Baire-1 functions is $\Sigma_{2}^{1}$ if the corresponding subset of $C^{\omega}$ is $\Sigma_{2}^{1}$.

For $\left\langle f_{i}\right\rangle \in C^{\omega}$, let $B_{\left\langle f_{i}\right\rangle}$ denote the following set of Baire-1 functions (cf. 1.1):

$\left\{h\right.$ : there is a subsequence of $\left\langle f_{i}\right\rangle$ which converges pointwise to $\left.h\right\}$.

Note that for any $\left\langle f_{i}\right\rangle, B_{\left\langle f_{i}\right\rangle}$ is $\Sigma_{2}^{1}$, uniformly. Define $\hat{E}$ as follows (cf. 2.5):

$\left\{\left\langle f_{i}\right\rangle \in C_{i}^{\omega}\right.$ : for every Baire-1 function $\left.h, h \in B_{\left\langle f_{i}\right\rangle}\right\}$.

Clearly $\hat{E}$ is $\boldsymbol{H}_{3}^{1}$. We have four questions about Baire-1 functions.

QUESTION 1. Is it true that for any set $S$ of Baire-1 functions, if $S$ is $\Sigma_{2}^{3}$ then there exists an $\left\langle f_{i}\right\rangle$ in $C^{(0)}$ such that $S=B_{\left\langle f_{i}\right\rangle}$ ?

Questron 2. Does there exist an $\left\langle f_{i}\right\rangle \in C^{\omega}$ such that $B_{\left\langle f_{i}\right\rangle}$ is the set of discontinuous Baire-1 functions?

QUisTion 3. Is $\widehat{E}$ ccmplete $\Pi_{3}^{1}$ ?

QUESTION 4. Is $\hat{E}$ not $\Sigma_{3}^{1}$ ?

An affirmative answer to Question $n$ implies an affirmative answer to Question $n+1(1 \leqslant n \leqslant 3)$. For $n=2$ we give an explanation, the other cases being trivial. Let $\left\langle g_{i}\right\rangle$ be a fixed sequence such that $B_{\left\langle g_{i}\right\rangle}$ is the discontinuous functions. For any $\left\langle f_{i}\right\rangle \in C^{\omega}$, let $\left\langle\hat{f_{i}}\right\rangle$ be the sequence: $\hat{f}_{2 i}=f_{i}, \hat{f}_{2 i+1}=g_{i}$. Then $B_{\left\langle\hat{f_{i}}\right\rangle}$ is the union of $A_{\left\langle f_{t}\right\rangle}$ and the set of discontinuous Baire-1 functions. So the map $\left\langle f_{i}\right\rangle \mapsto\left\langle\hat{f}_{i}\right\rangle$ reduces the set $E$ of 2.5 to $\hat{E}$. Since $E$ is complete, so is $\hat{E}$.

We have been able to prove the following weak version of a positive answer to Question 1. For $\left\langle f_{i}\right\rangle \in(C[0,2])^{\omega}$, let $\widetilde{B}_{\left\langle f_{i}\right\rangle}$ denote the following set of Baire-1 functions on $[0,1]$ :

$\left\{h\right.$ : there is a subsequence $\left\langle f_{n_{i}}\right\rangle$ of $\left\langle f_{i}\right\rangle$ such that $\left\langle f_{n_{i}}\right\rangle$ converges pointwise (on all of $[0,2]$ ) and for $\left.x \in[0,1], \lim _{i \rightarrow \infty} f_{n_{i}}(x)=h(x)\right\}$.

Then for every $\Sigma_{2}^{1}$ set $S$ of Baire-1 functions, there is an $\left\langle f_{i}\right\rangle$ such that $S=\widetilde{B}_{\left\langle f_{i}\right\rangle}$. This is enough to imply, as in 2.5 , that

$\hat{F}=\left\{\left\langle f_{i}\right\rangle \in C^{\omega}:\right.$ Some subsequence of $\left\langle f_{i}\right\rangle$ is pointwise convergent $\}$

is complete $\Sigma_{2}^{1}$, hence not $\boldsymbol{\Pi}_{2}^{1}$. (It follows from this, as in 2.5, that the $\boldsymbol{\Pi}_{1}^{1}$ set $\hat{\boldsymbol{G}} \subset C^{\omega}$ of pointwise convergent sequences is not $\Sigma_{1}^{1}$. However there is a simple direct proof that $\hat{G}$ is complete $\Pi_{1}^{1}$ - see [2]). 
2.12. For any $\left\langle f_{i}\right\rangle \in C^{o}$, let $K_{\left\langle f_{i}\right\rangle}$ denote the following subset of $C$ :

$\left\{h \in C\right.$ : there is a subsequence $\left\langle f_{n_{i}}\right\rangle$ of $\left\langle f_{i}\right\rangle$ such that $\left\langle f_{n_{i}}\right\rangle$ is uniformly bounded and $\left\langle f_{n_{i}}\right\rangle$ converges pointwise to $\left.h\right\}$.

This notion of convergence is weak convergence with respect to the Banach space $C[0,1]$. Again, for any $\left\langle f_{i}\right\rangle, K_{\left\langle f_{i}\right\rangle}$ is $\Sigma_{2}^{1}$, uniformly.

Question (Kechris). Is it true that for any $S \subset C$, if $S$ is $\Sigma_{2}^{1}$ then there exists an $\left\langle f_{i}\right\rangle \in C^{(i)}$ such that $S=K_{\left\langle f_{i}\right\rangle}$ ?

$\S 3$. Proof for $\Pi_{1}^{1}$ sets. In this section we prove the Main Theorem for the special case of $\Pi_{1}^{1}$ sets (Lemma 3.6, below).

We first establish some notation, following Moschovakis [12]. $X^{<(1)}$ is the set of all finite sequences from $X$. Finite sequences are denoted by Grcek letters $\sigma, \tau, \ldots$ $\sigma \prec \tau$ means that $\sigma$ is an initial segment of $\tau$, and similarly if $x$ is an infinite sequence $\sigma \prec x$ means that $\sigma$ is an initial segment of $x$. A tree $T$ on $X$ is a subset of $X^{<\omega}$ such that if $\sigma \prec \tau$ and $\tau \in T$ then $\sigma \in T$. The proof will involve trees on $\omega \times 2$; we identify a sequence in $(\omega \times 2)<\omega$ of length $n$ with two length $n$ sequences, one each from $\omega^{<\omega}$ and $2^{<\omega}$, and similarly for infinite sequences. Let $T$ be a tree on $\omega \times 2$. The body of the tree $T$, denoted $[T]$, is

$\left\{(x, y) \in \omega^{\omega} \times 2^{\omega}:\right.$ for all $\left.n,\left(x \uparrow^{-n} n, y \uparrow n\right) \in T\right\}$,

i.e., the set of all infinite branches through $T$.

Convergence of a sequence of functions always means pointwise convergence. Fix two families, $\left\{I_{\tau}^{\sigma}\right\}$ and $\left\{J_{\tau}^{\sigma}\right\}$, of closed subintervals of $[0,1]$, indexed by $(\sigma, \tau) \in(\omega \times 2)^{<\omega}$ and satisfying the following properties.

3.1. (a) $J_{\tau}^{\sigma} \subset$ Interior $\left(I_{\tau}^{\sigma}\right)$.

(b) If $(\sigma, \tau)$ is a proper initial segment of $\left(\sigma^{\prime}, \tau^{\prime}\right)$ then $I_{\tau^{\prime}}^{\sigma^{\prime}} \subset J_{\tau}^{\sigma}$.

(c) If $(\sigma, \tau)$ and $\left(\sigma^{\prime}, \tau^{\prime}\right)$ are incompatible then $I_{\tau}^{\sigma} \cap I_{\tau^{\prime}}^{\sigma^{\prime}}=\varnothing$. $\frac{1}{n+1}$.

For any $(y, z) \in\left(\omega^{\omega} \times 2^{\omega}\right)$, there is exactly one real number in

$$
\cap\left\{I_{\tau}^{\sigma}:(\sigma, \tau) \prec(y, z)\right\} ;
$$

let $r(y, z)$ denote the number.

3.2. For $n \in \omega$, define the set $Z_{n} \subset(\omega \times 2)^{<\omega}$ as follows. $(\sigma, \tau) \in Z_{n}$ if there exists a $k \in \omega$ such that:
(a) $\sigma=\left(i_{0}, i_{1}^{Y}, \ldots, i_{k-1}\right) \in \omega^{k}$
(b) $\tau=\left(j_{0}, j_{1}, \ldots, j_{k-1}\right) \in 2^{k}$,
(c) $i_{0}<i_{1}<i_{2}<\ldots<i_{k-1}$,
(d) $i_{k-1}=n$,
(e) $j_{k-1}=1$.

Note that $Z_{n}$ is finite and that the intervals $\left\{I_{\tau}^{\sigma}:(\sigma, \tau) \in Z_{n}\right\}$ are pairwise disjoint.
3.3. For any function $g \in C$, any $n \in \omega$, and any tree $T$ on $\omega \times 2$, let $f^{(g, n, T)}$ be the unique continuous function from $[0,1]$ into $R$ satisfying the following three conditions.

(a) If $x \notin \bigcup\left\{I_{\tau}^{\sigma}:(\sigma, \tau) \in Z_{n} \cap T\right\}$ then $f^{(g, n, T)}(x)=g(x)$.

(b) If $x \in \bigcup\left\{J_{\tau}^{\sigma}:(\sigma, \tau) \in Z_{n} \cap T\right\}$, then $f^{(g, n, T)}(x)=n$.

(c) On each interval of $U\left\{I_{\tau}^{\sigma} \backslash J_{\tau}^{\sigma}:(\sigma, \tau) \in Z_{n} \cap T\right\}, f^{(g, n, T)}$ is linear.

The first lemma examines a sequence of functions of the above form, and considers questions of convergence. (This lemma deals with a fixed sequence and does not consider convergence of its subsequences; however, it will later be applied to an arbitrary subsequence of a given sequence.) Let SI be the closed subspace of $\omega^{*}$ consisting of strictly increasing functions.

3.4. Lemma. Let $\left\langle g_{i}\right\rangle \in C^{()}$, let $w=\left(m_{0}, m_{1}, m_{2}, \ldots\right) \in \mathrm{SI}$, and let $T$ be a tree on $\omega \times 2$. Consider the sequence of functions:

$$
\left\langle f^{\left(g_{i}, m_{i}, T\right)}\right\rangle_{i \in \omega} .
$$

(a) If $\left\langle f^{\left(g_{1}, m_{i}, T\right)}\right\rangle$ converges then $\left\langle g_{i}\right\rangle$ converges and $\lim _{i \rightarrow \infty} f^{\left(g_{i}, m_{i}, T\right)}=\lim _{i \rightarrow \infty} g_{i}$.

(b) If $\left\langle f^{\left(\theta i, m_{l}, T\right)}\right\rangle$ does not converge and $\left\langle g_{i}\right\rangle$ does converge, then there is a $y \in \omega^{\omega}$ and $a z \in 2^{\omega}$ such that:

(i) $(y, z) \in[T]$

(ii) $w$ and $y$ have a common subsequence, i.e., there exist $v, \alpha, \beta \in \mathrm{SI}$ such that for all $j \in \omega, v(j)=w(\alpha(j))=y(\beta(j))$,

(iii) $\{j: z(j)=1\}$ is infinite.

(c) If there exists a $z \in 2^{\omega}$ such that

(i) $(w, z) \in[T]$,

(ii) $\{j: z(j)=1\}$ is infinite,

then $\left\langle f^{\left(g_{i}, m_{i}, T\right)}\right\rangle$ diverges.

Proof. Let $R=\left\{r(y, z): y \in \omega^{\omega}, z \in 2^{\omega}\right\}$. Two facts follow directly from the definitions (3.1 and 3.3).

(1) If $x \in[0,1]$ is not in $R$, then for all but finitely many $i \in \omega, f^{\left(g_{i}, m_{i}, T\right)}(x$ $=g_{i}(x)$.

(2) If $x \in R$, then for any $i$, either $f^{\left(g_{i}, m_{i}, T\right)}(x)=g_{i}(x)$ or $f^{\left(g_{i}, m_{i}, T\right)}(x)=m_{i}$.

(a) If $f^{\left(y_{i}, m_{i}, T\right)}(x)=m_{i}$ for infinitely many $i$, then $\left\langle f^{\left(g_{i}, m_{i}, T\right)}\right\rangle$ diverges. So (a) follows immediately from (1) and (2).

(b) Suppose that $\left\langle g_{i}\right\rangle$ converges and $\left\langle f^{\left(g_{i}, m_{i}, T\right)}\right\rangle$ does not. Then by (1) and (2), there exist $x \in[0,1], y \in \omega^{\omega}$, and $z \in 2^{\omega}$ such that $x=r(y, z)$ and for infinitely many $i$,

$$
f^{\left(g i, m_{i}, T\right)}(x)=m_{i} \neq g_{i}(x) .
$$

These infinitely many $i$ 's give us the subsequence. Formally, let $v \in \mathrm{SI}$ be a subsequence of $w$, say $v(j)=w(\alpha(j))$, such that for all $j \in \omega$,

$$
f^{\left(g_{\alpha(j)}, m_{\alpha(j)}, T\right)}(x)=m_{\alpha(j)}=w(\alpha(j))=v(j) \neq g_{\alpha(j)}(x) .
$$


By 3.3 (a), for any $j$ there must be a $\left(\sigma^{j}, \tau^{j}\right) \in Z_{v(j)} \cap T$ such that $x \in I_{\tau^{j}}^{\sigma^{j}}$. Since $x=r(y, z)$, clearly $\left(\sigma^{j}, \tau^{j}\right) \prec(y, z)$.

Let $k^{j}=$ length $\left(\sigma^{j}\right)$. By definition of $Z_{v(j)}$ (3.2), we have the following:

(i') $\left(\sigma^{j}, \tau^{j}\right) \in T$,

(ii') $\sigma^{j}\left(k^{j}-1\right)=v(j)$,

(iii') $\tau^{j}\left(k^{j}-1\right)=1$,

(iv') $\sigma^{j}(0)<\sigma^{j}(1)<\sigma^{j}(2)<\ldots<\sigma^{j}\left(k^{j}-1\right)$.

Since each $\sigma^{j}$ is an initial segment of $y$, clearly for any $j, j^{\prime} \in \omega, \sigma^{j}$ and $\sigma^{j^{\prime}}$ must be compatible. From this, (ii'), and (iv'), plus the fact that $v \in \mathrm{SI}$, it follows that $k^{j}$ is a strictly increasing function of $j$; that is, the inital segments $\left(\sigma^{j}, \tau^{j}\right)$ of $(y, z)$ keep getting longer. Hence (i') implies (i) of part (b), (ii') implies (ii), and (iii') implies (iii).

(c) Assume the hypothesis of (c). Let $x=r(w, z)$. Consider a $k \in \omega$ such that $z(k-1)=1$; there are infinitely many of these $k$ 's. Let $n=w(k-1)=m_{k-1}$, let $\sigma=w \uparrow k$ and let $\tau=z \uparrow k$. By definition of $Z_{n}(3.2),(\sigma, \tau) \in Z_{n}$. Then

$$
f^{\left(g_{k-1}, m_{k-1}, T\right)}(x)=m_{k-1}
$$

by definition of the function (see $3.3(\mathrm{~b})$ ). Since $w \in \mathrm{SI}, f^{\left(\theta_{l}, m_{l}, T\right)}(x)$ can be made arbitrarily large by choosing a large enough $i$ such that $z(i)=1$. So the sequence of reals $\left\langle f^{\left(g_{i}, m_{i}, T\right)}(x)\right\rangle$ diverges, hence the sequence of functions $\left\langle f^{\left(g l, m_{i}, T^{\prime}\right)}\right\rangle$ diverges.

3.5. LemmA. Let $Q \subset \omega^{\omega}$ be $\Sigma_{1}^{1}$. There is a tree $T$ on $\omega \times 2$ such that for all $y \in \omega^{\omega}$, $y \in Q \Leftrightarrow$ there exists $a z$ in $2^{\omega}$ such that $(y, z) \in[T]$ and $\{j: z(j)=1\}$ is infinite.

Proof. The usual representation for $\Sigma_{1}^{1}$ subsets of $\omega^{\omega}[12,2 \mathrm{~B}]$ gives a tree $U$ on $\omega \times \omega$ such that $Q$ is the projection of $[U]$ onto the first coordinate. Encode elements of $\omega^{\omega}$ by elements of $2^{\omega}$ in the following way: $z \in 2^{\omega}$ is a code iff infinitely many of its coordinates are 1 , and it encodes $\hat{z} \in \omega^{\omega}$ where

$\hat{z}(n)=$ the number of 0 's between the $n$th and the $(n+1)^{\text {st }} 1$ in $z$.

Let $T$ be the tree on $\omega \times 2$ that corresponds to $U$ under this coding; that is, $(\sigma, \tau) \in T$ iff there exists a $y \in \omega^{\omega}$ and a $z \in 2^{\omega}$ such that $(\sigma, \tau) \prec(y, z)$ and $z$ is a code and $(y, \hat{z}) \in[U]$. It is easy to see that $T$ satisfies the lemma.

(There is a reason for representing $\Sigma_{1}^{1}$ sets via trees on $\omega \times 2$, rather than using the customary trees on $\omega \times \omega$. It is necessary to work with 2 rather than $\omega$ in defining the sets $Z_{n}$ in 3.2, since if $\omega$ is used then $Z_{n} \cap T$ may be infinite. If $Z_{n} \cap T$ is infinite, then in defining the function $f^{(g, n, T)}$, in 3.3 , there will be infinitely many intervals, hence there will be limit points, hence $f^{(g, n, T)}$ will not be continuous.)

3.6. LEMMA. For any $P \subset C$, if $P$ is $\Pi_{1}^{1}$ then there exists an $\left\langle f_{i}\right\rangle$ in $C_{1}^{\infty}$ such that $P=A_{\left\langle f_{i}\right\rangle}$.

Proof. Let $p_{0}, p_{1}, p_{2}, \ldots$ be a fixed sequence of functions which is dense in $C$, e.g., the polynomials with rational coefficients. Define $Q \subset \omega^{\omega}$ as follows:

$$
y \in Q \Leftrightarrow\left[y \in \operatorname{SI} \&(\exists h \in C)\left(h \notin P \& \text { for a.e. } x \in[0,1], \lim _{i \rightarrow \infty} p_{y(i)}(x)=h(x)\right)\right] .
$$

The pointset $Q$ is $\Sigma_{1}^{1}$. This is proved by the methods of [12,1C and 1E], i.e., quantifier-counting. That is, the complement of $P$ is $\Sigma_{1}^{1}$ and the pointclass of $\Sigma_{1}^{1}$ sets is closed under all the operations used to define $Q$, so $Q$ is $\Sigma_{1}^{1}$. We are using one nontrivial closure property of $\Sigma_{1}^{1}$ (in addition to the trivial ones), namely the fact that $\Sigma_{1}^{1}$ is closed under quantification of the form "for a.e. $x \in[0,1]$ ", where almost every rcfers to Lebesgue measure on $[0,1]$; this is a theorem of Tanaka [16] - see also Sacks [15] and Kechris [7]. (The motivation for this definition is that we would really like to define $Q$ to consist of those $y$ in SI such that the subsequence $\left\langle p_{y(i)}\right\rangle$ of $\left\langle p_{i}\right\rangle$ converges pointwise to an element of $C \backslash P$; the problem is that this is not $\Sigma_{1}^{1}$, and we need a $\Sigma_{1}^{1}$ set. The only reason for introducing measure at all is to get around this problem.)

Since $Q$ is $\Sigma_{1}^{1}$, by Lemma 3.5, there is a tree $T$ on $\omega \times 2$ such that for all $y \in \omega^{\omega}$,

3.7. $y \in Q \Leftrightarrow$ there exists a $z$ in $2^{\omega}$ such that $(y, z) \in[T]$ and $\{j: z(j)=1\}$ is infinite.

Fix such a $T$. For all $n \in \omega$, let $f_{n}=f^{\left(p_{n}, n, T\right)}$ (see 3.3 for definition). We will prove below that $P=A_{\left\langle f_{i}\right\rangle}$.

$P \subset A_{\left\langle f_{i}\right\rangle}$. Let $g \in P$. Let $\left\langle p_{n_{t}}\right\rangle$ be a subsequence of $\left\langle p_{i}\right\rangle$ which converges to $g$. We show that $\left\langle f_{n_{i}}\right\rangle$ converges to $g$. Assume that this is not so. Then by Lemma 3.4 (a), $\left\langle f_{n_{i}}\right\rangle$ does not converge. So by $3.4(\mathrm{~b})$, there is a $y \in \omega^{\omega}$ and a $z \in 2^{\omega}$ such that:

(i) $(y, z) \in[T]$

(ii) $\left\langle n_{i}\right\rangle$ and $y$ have a common subsequence,

(iii) $\{j: z(j)=1\}$ is infinite.

From (i), (iii) and 3.7 we conclude that $y \in Q$. By definition of $Q$, there is an $h \in C$ such that $h \notin P$ and for a.e. $x, \lim _{i \rightarrow \infty} p_{y(i)}(x)=h(x)$. By (ii), the sequences $\left\langle p_{n_{i}}\right\rangle$ and $\left\langle p_{y(i)}\right\rangle$ have a common subsequence, hence for any point $x \in[0,1]$, if $\left\langle p_{n_{i}}\right\rangle$ and $\left\langle p_{y(i)}\right\rangle$ both converge at $x$, then they must both converge to the same value. So for a.e. $x, g(x)=h(x)$. But $g$ and $h$ are both continuous functions, so $g=h$. This contradicts the fact that $g \in P$ and $h \notin P$.

$A_{\left\langle f_{i}\right\rangle} \subset P$. Let $\left\langle f_{m i}\right\rangle$ be a subsequence of $\left\langle f_{i}\right\rangle$, let $h \in C$, and suppose that $\left\langle f_{n_{1}}\right\rangle$ converges to $h$. We must show that $h \in P$. By Lemma 3.4 (a), $\left\langle p_{m}\right\rangle$ converges to $h$. Let $w=\left(n_{0}, n_{1}, n_{2}, \ldots\right)$. Now suppose that $h \notin P$. Then by definition of $Q$, $w \in Q$. So by 3.7 , there exists a $z \in 2^{\omega}$ such that $(w, z) \in[T]$ and $z$ has infinitely many 1 's. That is, $w$ satisfies the hypothesis of Lemma $3.4(\mathrm{c})$; therefore $\left\langle f_{n_{i}}\right\rangle$ diverges, contrary to assumption.

§ 4. Proof for $\Sigma_{2}^{1}$ sets. Lemma 3.6 and its proof can be generalized from $C$ to a large class of spaces. In this section of the paper we give the generalization, and from it derive the Main Theorem (1.2). 
Consider two real numbers $a$ and $b$, with $a<b$, and a nonempty closed set $F$ in $C[a, b]$. Then $F$ (topologized as a subspace of $C[a, b])$ and $F^{\omega}$ are Polish spaces. Call $F$ suitable if there exist two reals $c$ and $d$, with $a<c<d<b$, satisfying the following property: For any functions $f^{\prime}$ and $g$ in $C[a, b]$, if $g \in F$ and for every $x$ in $([a, b] \backslash[c, d]), f(x)=g(x)$, then $f \in F$. For $\left\langle f_{i}\right\rangle \in F^{\omega}$, let $A_{\left\langle f_{i}\right\rangle}^{F}$ denote the following subset of $F$ :

$\left\{h \in F\right.$ : there is a subsequence of $\left\langle f_{l}\right\rangle$ which converges pointwise to $h$ \}.

This definition is, of course, the analog of 1.1 for the space $F$.

4.1. Lemma. Let $a, b \in R$, with $a<b$, and let $F \subset C[a, b]$ be a suitable closed set. For any $P \subset F$, if $P$ is $\Pi_{1}^{1}$ then there exists an $\left\langle f_{i}\right\rangle$ in $F^{(1)}$ such that $P=A_{\left\langle s_{i}\right\rangle}^{F}$.

The proof of Lemma 4.1 is essentially the same as the proof of Lemma 3.6 given in $\S 3$. There is one extra detail: Make sure that the intervals $l_{\tau}^{\sigma}$ of 3.1 all lie within $[c, d]$, where $c$ and $d$ are as in the definition of suitable. (Thus the coding up of the tree into the functions all takes place inside $[c, d]$.) The only obstacle to generalizing $\$ 3$ to arbitrary closed subsets of $C[a, b]$ is that the new functions $f^{(g, n, T)}$ constructed in 3.3 may not be in that closed set; suitability guarantees that this problem will not occur.

Now we fix an $F$. Let $F$ be the following subset of $C[0,2]$ :

$\{f \in C[0,2]: f(2) \in[0,1]$ and $f \uparrow[1,2]$ is linear $\}$.

Note that this $F$ is closed and suitable, so Lemma 4.1 is applicable to it.

Next consider the spaces $D=(C[0,1] \times[0,1])$ and $D^{\omega}$. We say that a sequence $\left\langle g_{i}, y_{i}\right\rangle \in D^{\omega}$ converges to $(h, y)$ if $\left\langle g_{i}\right\rangle$ converges pointwise to $h$ and $\left\langle y_{i}\right\rangle$ converges to $y$. For any $\left\langle g_{i}, y_{i}\right\rangle \in D^{\omega}$, let $B_{\left\langle g_{i}, y_{i}\right\rangle}$ denote the following subset of $D$ :

$\left\{(h, y) \in D\right.$ : there is a subsequence of $\left\langle g_{i}, y_{i}\right\rangle$ which converges to $\left.(h, y)\right\}$.

4.2. Lemma. For any $P \subset D$, if $P$ is $\Pi_{1}^{1}$ then there exists $a\left\langle g_{i}, y_{i}\right\rangle \in D^{(\omega)}$ such that $P=B_{\left\langle g_{i}, y_{i}\right\rangle}$.

Proof. Let $P \subset D$ be $\Pi_{1}^{1}$. Let $P^{*}$ be the subset of $F$ defined as follows.

$$
P^{*}=\{f \in F:(f \uparrow[0,1], f(2)) \in P\} .
$$

There is a homeomorphism from $D$ onto $F$ which maps $P$ to $P^{*}$, hence $P^{*}$ is also $\Pi_{1}^{1}$. So by Lemma 4.1, there is an $\left\langle f_{i}\right\rangle \in F^{\omega}$ such that $P^{*}=A_{\left\langle f_{i}\right\rangle}^{F}$. Let $g_{i}=f_{i} \uparrow[0,1]$ and let $y_{i}=f_{i}(2)$. Then it is easy to see that $B_{\left\langle q_{i}, y_{i}\right\rangle}=P$.

We can now lift the representation theorem from $\Pi_{1}^{1}$ sets to $\Sigma_{2}^{1}$ sets.

Proof of Theorem 1.2. Let $S \subset C$ be a $\Sigma_{2}^{1}$ set. Then there is a $\Pi_{1}^{1}$ set $P \subset(C \times[0,1])=D$ such that $S$ is the projection of $P$, that is, for all $h \in C$ :

$h \in S \Leftrightarrow$ there exists a $y \in[0,1]$ such that $(h, y) \in P$.

(The official definition of $\Sigma_{2}^{1}$ given in this paper is that a subset of $X$ is $\Sigma_{2}^{1}$ if it is the projection of a $\Pi_{1}^{1}$ set in $X \times Y$ for some $Y$. But all uncountable Polish spaces are Borel isomorphic, so without loss of generality, the space $Y$ may be taken to be $[0,1]$. Moschovakis [12] defines a subset of $X$ to be $\Sigma_{2}^{1}$ if it is the projection of a $\boldsymbol{\Pi}_{1}^{1}$ set in $X \times \omega^{\omega}$; the same remark applies to this definition.)

By Lemma 4.2 , there is a sequence $\left\langle f_{i}, y_{i}\right\rangle \in D^{\omega}$ such that $P=B_{\left\langle f_{i}, y_{i}\right\rangle}$. We show below that $S=A_{\left\langle f_{i}\right\rangle}$, and thus prove Theorem 1.2.

$S \subset A_{\left\langle f_{i}\right\rangle}$. Let $h \in S$. Then there is a $y \in[0,1]$ such that $(h, y) \in P$. Since $P$ is $B_{\left\langle f_{i}, y_{i}\right\rangle}$ there is a subsequence $\left\langle f_{n_{i}}, y_{n_{i}}\right\rangle$ of $\left\langle f_{i}, y_{i}\right\rangle$ which converges to $(h, y)$. Therefore $\left\langle f_{n_{t}}\right\rangle$ converges to $h$, and hence, $h \in A_{\left\langle f_{i}\right\rangle}$.

$A_{\left\langle f_{i}\right\rangle} \subset S$. Suppose that $h \in A_{\left\langle f_{i}\right\rangle}$. Then by definition of $A_{\left\langle f_{i}\right\rangle}$, there is a subsequence $\left\langle f_{n_{i}}\right\rangle$ of $\left\langle f_{i}\right\rangle$ which converges to $h$. Consider the corresponding sequence $\left\langle y_{n i}\right\rangle$ of points in $[0,1]$. By compactness it has a convergent subsequence - call it $\left\langle y_{m_{i}}\right\rangle$ - and let $y$ be the limit of $\left\langle y_{m_{i}}\right\rangle$. Now $(h, y)$ is a point in $C \times[0,1]=D$ and the sequence $\left\langle f_{m_{l}}, y_{m_{i}}\right\rangle \in D^{\omega}$ converges to $(h, y)$; so by definition of $B_{\left\langle f_{i}, y_{i}\right\rangle}$, $(h, y) \in B_{\left\langle f_{i}, y_{i}\right\rangle}$. Since $B_{\left\langle f_{i}, y_{i}\right\rangle}=P$ and $S$ is the projection of $P, h \in S$.

\section{References}

[1] F. Bagemihl and J. E. Mc Millan, Characterization of the sets of angular and global convergence, and of the sets of angular and global limits, of functions in a half-plane, Fund. Math. 59 (1966), 177-187.

[2] H. Becker, Some examples of Borel-inseparable pairs of coanalytic sets, Mathematika 33 (1986) 72-79.

[3] W. Hurewicz, Zur Theorie der analytischen Mengen, Fund. Math. 15 (1930), 4-17

[4] T. J. Jech, Set Theory, Academic Press, New York 1978.

[5] R. Kaufman, Lipschitz spaces and Suslin sets, J. Funct. Anal. 42 (1981), 271-273.

[6] - Representation of Sustin sets by operators, Integral Equations Operator Theory 7 (1984), 808-814.

[7] A. S. Kechris, Measure and category in effective descriptive set theory, Ann. Math. Logic 5 (1973), 337-384.

[8] A.S. Kechris and W. H. Woodin, Ranks of differentiable functions, Mathematika 33 (1986), 252-287.

[9] A. Lévy, Definability in axiomatic set theory, I, in Logic, Methodology and Philosophy of Science, Y. Bar-Hillel (Ed.), North-Holland, Amsterdam 1965, 127-151.

[10] G. G. Lorentz and K. Zeller, Series rearrmgements and analytic sets, Acta Math. 100 (1958), $149-169$.

[11] S. Mazurkiewicz, Über die Mcnge der differenzierbaren Funktionen, Fund. Math. 27 (1936), 244-249.

[12] Y. N. Moschovakis, Descriptive Set Theory, North-Holland, Amsterdam 1980.

[13] T. Nishiura, Uncountuble-order sets for radial-limit functions, Real Anal. Exchange 10 (1984-85), 50-53.

[14] G. Poprougénko, Sur l'analyticité des ensembles (A), Fund. Math. 18 (1932), 77-84.

[15] G. E. Sacks, Measure-theoretic uniformity in recursion theory and set theory, Trans. Amer. Math. Soc. 142 (1969), 381-420. 
[16] H. Tanaka, A basis result for $\Pi_{1}^{1}$-sets of positive measure, Comment. Math. Univ. of St. Paul $16,(1967-68), 115-127$.

[17] Z. Zalcwasser, Sur une propriété du champ des fonctions continues, Studia Math. 2 (1930), 63-67.

DEPT. OF MATH.

UNIVERSITY OF SOUTH CAROLINA

Columbia, South Carolina 29208

Receired 19 August 1985

\section{Solution of Kuratowski's problem on function having the Baire property, $I$.}

by

Ryszard Frankiewicz (Warszawa) and Kenneth Kunen (Madison, Wis.)

Abstract. In this paper it is proved: $\mathrm{ZFC}+$ "there is measurable cardinal" is equiconsisten with $\mathrm{ZFC}+$ "there is a Baire metric space $X$, a metric space $Y$, and a function $f: X \rightarrow Y$ having the Baire property such that there is no meager set $F \subseteq X$ for which $f \mid X \backslash F$ is continuous".

In $1935 \mathrm{~K}$. Kuratowski [11] posed the following problem: whether a function $f: X \rightarrow Y$ having the Baire property, where $X$ is completely metrizable and $Y$ is metrizable, is continuous apart from a meager set (cf. P. 6 [12]).

In this paper it will be proved:

THEOREM. The following theories are equiconsistent:

(1) $\mathrm{ZFC}+\exists$ measurable cardinal

(2) ZFC + there is a complete metric space $X$, a metric space $Y$, and a function $f: X \rightarrow Y$ having the Baire property such that there is no meager set $F \subseteq X$ for which $f \mid X \backslash F$ is continuous;

(3) $\mathrm{ZFC}+$ there is a Baire metric space $X$, a metric space $Y$, and a function $f: X \rightarrow Y$ having the Baire property such that there is no meager set $F \subseteq X$ for which $f \mid X \backslash F$ is continuous.

1. Definitions and the basic facts. Let $X$ be a topological space, and $A \subseteq X$. The set $A$ is said to have the Baire property if

$$
A=\left(G \backslash P_{1}\right) \cup P_{2} \text {, }
$$

where $G$ is open and $P_{1}, P_{2}$ are meager sets (for basic facts see Kuratowski [10]). A map $f: X \rightarrow Y$ has the Baire property iff for each open set $V \subseteq Y, f^{-1}(V)$ has the Baire property.

$1.1 \mathrm{In}$ [4] the equivalence of the following statements has been proved: Let $X, Y$ be metric

(i) for each subspace $X^{*}=G \backslash F$ of $X$, where $G$ is a nonempty open set and $F$ is a meager set and for each partition $\mathscr{F}$ of $X^{*}$ into meager sets, there is a family $\mathscr{F}^{\prime} \subseteq \mathscr{F}$ such that $\mathscr{F}^{\prime}$ does not have the Baire property.

3 - Fundamenta Mathematicae CXxvirr. 3 